\title{
Left ventricular hypertrophy caused by arterial hypertension and degenerative aortic stenosis: How useful ${ }^{123}$ I-mIBG is
}

\author{
Giuseppe De Vincentis, MD, PhD, ${ }^{\mathrm{a}}$ and Viviana Frantellizzi, $M D, \mathrm{PhD}^{\mathrm{a}}$ \\ a Department of Radiological Sciences, Oncology and Anatomical Pathology, Sapienza Univer- \\ sity of Rome, Rome, Italy
}

Received Jul 15, 2020; accepted Jul 16, 2020

doi: $10.1007 / \mathrm{s} 12350-020-02295-\mathrm{x}$

\section{See related article, pp. 337-347}

The study of the activity of the sympathetic nervous system (SNS) is gaining increasing importance in several areas of cardiac pathology. In cardiomyopathy patients (of ischemic origin or not), myocardial assessment of early and late ${ }^{123} \mathrm{I}-m \mathrm{IBG}$ distribution, is widely recognized as a very powerful survival predictor. ${ }^{1,2}$ Furthermore, in general, we can affirm that chronic heart failure $(\mathrm{CHF})$ patients have overactivation of the reninangiotensin-aldosterone and SNS, which creates a vicious circle of remodeling and an accompanying worse prognosis. $^{3-5}$ Since the early 1970s, it is known that the SNS, as a compensatory mechanism, is hyperactivated during exercise in patients with even a slight degree of valvular heart disease compared to normal subjects. ${ }^{6}$ In western countries, aortic stenosis (AS) is the most common heart valve disease. Symptomatic patients have the wrong prognosis, as $75 \%$ of them die within 3 years of the symptom's onset. The standard treatment for patients with severe symptomatic AS is the surgical replacement of the aortic valve. However, 33\% of all patients aged 75 or older are not eligible for surgery. Transcatheter aortic valve implantation (TAVI) is an emerging therapeutic option in patients with AS at high risk for surgery. ${ }^{7}$ Contrary to the traditional open heart, the TAVI procedure is minimally invasive when performed through transfemoral access and can be

Reprint requests: Giuseppe De Vincentis, MD, PhD, Department of Radiological Sciences, Oncology and Anatomical Pathology, Sapienza University of Rome, Rome, Italy; giuseppe.devincentis@uniroma1.it

J Nucl Cardiol 2022;29:348-9.

$1071-3581 / \$ 34.00$

Copyright (c) 2020 American Society of Nuclear Cardiology. performed with light sedation and without cardiopulmonary bypass. According to current guidelines, invasive treatment is delayed until the development of symptoms or impairment of left ventricular ejection fraction (LVEF). " However, this "wait for symptoms", strategy is often ineffective or even harmful to patients, and it is widely believed that increased SNS activity is a sign of disease progression. ${ }^{10}$ An increase in SNS activity can trigger or exacerbate many of the pathophysiological features associated with SA such as cardiac hypertrophy. Besides, sympathetic stimulation increases the heart rate, with all the consequences that this entails. Therefore, an increase in SNS activity may represent an important risk factor for the progression and the outcome of patients with AS. In the article "Cardiac sympathetic dysfunction in left ventricular hypertrophy caused by arterial hypertension and degenerative aortic stenosis" by Liga et al., published in this issue of the Journal of Nuclear Cardiology, authors focus their interest "to evaluate the possible independent contribution of HT and AS on myocardial SNS activity' at the early stage of the disease. ${ }^{11}$ The study aimed to assess the regional myocardial innervation/perfusion in hypertensive patients with degenerative AS undergoing TAVI. Moreover, they evaluate the impact of successful left ventricular (LV) unloading by TAVI after 6 months. The study contains many innovative elements, as patients with severe AS and severe left ventricle hypertrophy $(\mathrm{LVH})$ at low-intermediate surgical risk, submitted to TAVI, were studied. This aspect should be underlined, as the surgical aortic valve replacement may damage cardiac autonomic nerves, and the surgical procedure itself may influence the sympathetic nerve activity. On the opposite, TAVI is less invasive than open surgery and therefore may minimally affect cardiac autonomic nerves. An observation of great physiological relevance is represented by the fact that no significant difference in regional ${ }^{123} \mathrm{I}-m \mathrm{IBG}$ washout (WO) was 
observed both in patients and in controls. In this paper, the regional ${ }^{123} \mathrm{I}-m \mathrm{IBG}$ washout was calculated on a myocardial segment basis as follows: regional $\mathrm{WO}=$ $100 \times[$ (mean early segmental counts - mean delayed segmental counts)/mean early segmental counts]. These data express that in the early phase of the pathology the adrenergic system shows a conserved function, even in the presence of a quantitative reduction of the functional units. Taking into account the burgeoning literature on the role of ${ }^{123} \mathrm{I}-m \mathrm{IBG}$ in $\mathrm{CHF}$, the results presented by the authors must be weighed based on exclusion criteria, among which figures a depressed LVEF $(<50 \%)$. It looks very intriguing that a higher LV mass index was the only independent predictor of impaired LV sympathetic innervation. As the Authors affirm, "the major and original findings of this study is the strong, independent association of LVH with impaired LV adrenergic innervations in essential hypertension (HT) patients with AS or not, and second, the missing change in ${ }^{123} \mathrm{I}-m \mathrm{IBG}$ uptake in patients in whom TAVI procedure had eliminated the increased trans-valvular gradient' '. Which came first, the chicken or the egg? In the literature appears clear how several studies want to clarify the role of the relationship between AS and sympathetic tone in the development of cardiac hypertrophy. A strength of the Liga et al. study is that, by eliminating many confounding factors in the interpretation of the cardiac study using ${ }^{123} \mathrm{I}-m \mathrm{IBG}$, they demonstrate how in "elder patients with long-standing HT, with or without AS, the severity of LVH is the major determinant of cardiac sympathetic abnormalities'. Of course, some limitations affect this study. The number of patients is limited, and the incidence of diabetic subjects is significant. In our opinion, in future studies, the knowledge base could be enriched by calculating the semiquantitative parameters, classically used in the cardiac study using ${ }^{123} \mathrm{I}-m \mathrm{IBG}$, such as early and late heart to mediastinum $(\mathrm{H} / \mathrm{M})$ ratio and tracer washout examples, deriving them directly from the data obtained with the tomographic acquisition, as suggested by Shibutani et al. ${ }^{12}$ This could make the diagnostic procedure more tolerable for the patient without affecting its reliability. However, what appears relevant, is the methodological approach proposed by Liga et al., that allows adding knowledge on the origin and evolution of myocardial hypertrophy present in AS, but above all how it is possible, through molecular diagnostics, what is the ${ }^{123} \mathrm{I}-m \mathrm{IBG}$ cardiac study, to obtain personalized procedures in the therapeutic decision and in the longterm treatment of these patients, which in this way is optimized on the individual subject.

\section{Disclosures}

Giuseppe De Vincentis and Viviana Frantellizzi have no disclosures.

\section{References}

1. Carrió I, Cowie MR, Yamazaki J, Udelson J, Camici PG (2010) Cardiac sympathetic imaging with $\mathrm{mIBG}$ in heart failure. JACC Cardiovasc Imaging 3:92-100

2. De Vincentis G, Frantellizzi V, Fedele F, Farcomeni A, Scarparo $\mathrm{P}$, Salvi N et al (2018) Role of cardiac 123I-mIBG imaging in predicting arrhythmic events in stable chronic heart failure patients with an ICD. J Nucl Cardiol 26:1188-1196

3. Cohn JN, Levine TB, Olivari MT, Garberg V, Lura D, Francis GS et al (1984) Plasma norepinephrine as a guide to prognosis in patients with chronic congestive heart failure. N Engl J Med 311:819-823

4. Kawaguchi H, Kitabatake A (1995) Renin-angiotensin system in failing heart. J Mol Cell Cardiol 27:201-209

5. Mangieri E, Tanzilli G, De Vincentis G, Barillà F, Remediani S, Acconcia MC et al (2006) Slow coronary flow and stress myocardial perfusion imaging. Different patterns in acute patients. J Cardiovasc Med (Hagerstown) 7:322-327

6. Tönnesen KH, Uhrenholdt A, Pedersen-Bjergaard O (1974) Hand blood flow and cardiac functional response to severe exercise in normal subjects and patients with valvular heart disease. Scand $\mathbf{J}$ Clin Lab Invest 34:123-131

7. Dumonteil N, Vaccaro A, Despas F, Labrunee M, Marcheix B, Lambert E et al (2013) Transcatheter aortic valve implantation reduces sympathetic activity and normalizes arterial spontaneous baroreflex in patients with aortic stenosis. JACC Cardiovasc Interv 6:1195-1202

8. Vahanian A, Alfieri O, Andreotti F, Antunes MJ, Barón-Esquivias G, Baumgartner H et al (2012) Guidelines on the management of valvular heart disease (version 2012). Eur Heart J 33:2451-2496

9. Nishimura RA, Otto CM, Bonow RO, Carabello BA, Erwin JP, Guyton RA et al (2014) 2014 AHA/ACC guideline for the management of patients with valvular heart disease: A report of the American College of Cardiology/American Heart Association Task Force on Practice Guidelines. J Am Coll Cardiol 63:e57-185

10. Rizas KD, Zuern CS, Bauer A (2017) Periodic repolarization dynamics in patients with moderate to severe aortic stenosis. $\mathrm{J}$ Electrocardiol 50:802-807

11. Liga R, Gimelli A, De Carlo M, Marzullo P, Pedrinelli R, Petronio AS (2020) Cardiac sympathetic dysfunction in left ventricular hypertrophy caused by arterial hypertension and degenerative aortic stenosis. J Nucl Cardiol

12. Shibutani T, Nakajima K, Yoneyama H, Konishi T, Okuda K, Onoguchi M et al (2020) The utility of heart-to-mediastinum ratio using a planar image created from IQ-SPECT with Iodine-123 meta-iodobenzylguanidine. J Nucl Cardiol. https://doi.org/10.100 7/s12350-020-02081-9

Publisher's Note Springer Nature remains neutral with regard to jurisdictional claims in published maps and institutional affiliations. 